\title{
Management of Subtalar Fractures-Dislocations at Ségou Hospital in Mali: A 7 Cases Series
}

\author{
Mahamadou Diallo" ${ }^{*}$, Abdoul Kadri Moussa², Layes Toure3, Louis Traoré2, \\ Cheick Oumar Sanogo ${ }^{4}$, Terna Traore ${ }^{3}$, Seydina Alioune Beye ${ }^{5}$, Abdrahamane Toure ${ }^{1}$, \\ Making Mounkoro', Mahamadou Kane' ${ }^{1}$, Tiéman Coulibaly ${ }^{2}$
}

${ }^{1}$ Orthopedic-Trauma Service, Nianankoro Fomba Hospital, Ségou, Mali

${ }^{2}$ Orthopedic-Trauma Service, Gabriel Touré Hospital, Bamako, Mali

${ }^{3}$ Orthopedic-Trauma Service, Sikasso Hospital, Sikasso, Mali

${ }^{4}$ Orthopedic-Trauma Service, Kati Hospital, Kati, Mali

${ }^{5}$ Anesthesia-Resuscitation Service, Nianankoro Fomba Hospital, Ségou, Mali

Email: *diallo_mohamed67@yahoo.fr

How to cite this paper: Diallo, M., Moussa, A.K., Toure, L., Traoré, L., Sanogo, C.O., Traore, T., Beye, S.A., Toure, A., Mounkoro, M., Kane, M. and Coulibaly, T. (2020) Management of Subtalar Fractures-Dislocations at Ségou Hospital in Mali: A 7 Cases Series. Surgical Science, 11, 51-60.

https://doi.org/10.4236/ss.2020.114007

Received: March 3, 2020

Accepted: April 7, 2020

Published: April 10, 2020

Copyright $\odot 2020$ by author(s) and Scientific Research Publishing Inc. This work is licensed under the Creative Commons Attribution International License (CC BY 4.0).

http://creativecommons.org/licenses/by/4.0/

\begin{abstract}
Introduction: Subtalar or peritalian dislocation is rare; it represents $15 \%$ of peritalian injuries. The aim of this study was to describe the radioclinical and therapeutic characteristics and to assess the functional result. Patients and Methods: This study is about a continuous descriptive and prospective study over a period of 5 years. It has been conducted from March 2013 to February 2018 at the Ségou Hospital, a second referral hospital in Mali. The functional results were evaluated by the score of the American Orthopedic Foot and Ankle Society (AOFAS). Results: Seven cases of subtalar dislocation fractures were diagnosed in six male patients with an average age of 38 years (36 to 47 years old). In five cases the etiology of the trauma was a motorcycle accident and a fall from height. The lesion was bilateral in one case. The average time to care was 3 hours ( 1 to 9 hours). There was one case of open dislocation fracture with talus enucleation. The dislocation was medial in 6 cases and lateral in one case. It was pure in three cases. The treatment was orthopedic in 2 cases and surgical in 5 cases. The post-operative complications were complicated by an infection of the operative site in one case. The average length of hospital stay was 4 days. The functional result was excellent in 2 cases; good in 2 cases, fair in 2 cases and poor for 1 case. Conclusion: Subtalar dislocation is a rare and serious lesion of the posterior tarsus. The long-term prognosis depends on the earliness of treatment and the severity of the associated injuries.
\end{abstract}

\section{Keywords}

Fracture, Dislocation, Subtalar, Prognosis, Treatment 


\section{Introduction}

Subtalar or peritalian dislocations affect the talo-calcaneal and talo-navicular joints; the tibio-talian and calcaneo-cuboid joints being intact [1] [2]. The stability of the subtalar joint is ensured by powerful ligaments. Most of these dislocations occur in a context of high-energy trauma such as falls, sports, motorcycle or automobile accidents [3]. It is rare in routine trauma practice, represents $1 \%-2 \%$ of all dislocations and $15 \%$ of peritalian lesions [4]. Ankle deformation related to dislocation is usually obvious. In the context of polytrauma, dislocation can go unnoticed. Computed tomography is of great help, confirms the diagnosis and assesses the associated osteocartilaginous lesions [1] [2] [5] [6].

Pure dislocation reduced and immobilized early has a good prognosis. It is associated with fracture and other peritalian lesions in $60 \%$ of cases. Ignorance of these associated lesions can quickly lead to the installation of pain in the peritalian joint [7] [8]. The objective of this work consists in describing the radio-clinical and therapeutic characteristics and in evaluating the result functional of subtalar dislocations.

\section{Patients and Methods}

It is a continuous descriptive and prospective study over a period of 5 years extending from March 2013 to February 2018. It was carried out in the orthopedics and traumatology department of the Ségou Hospital which is a 2nd Reference Hospital of Mali. The hospital's medical imaging department does not have a CT scan. The study population involved patients received and treated for dislocation or subtalar fracture-dislocation during the study period. Each patient had a medical file in which the clinical and radiological follow-up was mentioned. The average follow-up was 18 months.

We have adopted the Malgaigne classification [9] which allows us to distinguish 4 varieties of dislocation:

- Medial subtalar dislocation: $50 \%$ to $90 \%$ of subtalar dislocations;

- Less frequent lateral subtalar dislocation;

- The subtalar dislocations in the anterior and posterior variety are exceptional.

\subsection{Therapeutic Method}

Reduction of the dislocation was done in an emergency. In closed dislocations the reduction, often orthopedic, was carried out under general anesthesia in order to obtain optimal muscle relaxation. The reduction in the medial variety was obtained by plantar flexion of the foot with traction of the forefoot to release the head from the slope, followed by a varus maneuver to raise the foot below the slope. The open reduction had been indicated in irreducible and open dislocations. In these open dislocations antibiotic therapy based on amoxicillin-clavulanic acid was administered upon admission of the patient. At the same time, trimming, reduction of dislocation and skewering were performed. Functional rehabilitation began immediately post-operatively and was continued until the ankle and foot function recovered. 


\subsection{Assessment Method}

The results were assessed according to clinical, radiological and functional criteria. The average decline was 18 months with extremes of 12 to 36 months. The functional results were evaluated by the score of the American Orthopedic Foot and Ankle Society (AOFAS) [10]:

- Pain (40 points)

- No

40

- Medium, occasional

30

- Moderate, daily 20

- Severe, constant

- Function (50 points)

Activity limitation

- No limitation, no help

- No limitation of daily activities on the other hand sports limitation, no help

- Daily limitation, 1 cane

- Severe limitation, canes, armchair

Distance, walking distance

- $1 \mathrm{~km}$

- 400 to 600 meters

200 to 400 meters

- Less than 200 meters

Surface condition

- All types of terrain $\quad 5$

- Difficulty on inclined terrain or stairs 3

- Severe difficulty on any terrain

Gait analysis

- Normal

- Moderate disorder

- Frank lameness

Mobility in flexion and extension

- Normal or $>30^{\circ}$

- $15^{\circ}$ to $30^{\circ}$

- Less than $15^{\circ}$

Hindfoot mobility (inversion and eversion)

- $75 \%$ to $100 \%$ of normal

- $27 \%$ to $75 \%$ (moderate)

- $\quad<25 \%$ stiffness

Ankle stability

- Stable

- Unstable

- Alignment (10 points)

- Good, plantigrade foot, ankle and rear foot oriented 
- Low misalignment, without symptoms 5

- Bad, not plantigrade foot, severe misalignment, symptomatic 0

Depending on the score the result is judged:

- Excellent: score between 90 and 100

- Good: score between 80 and 90

- Fair: score between 70 and 80

- Poor: score less than 70

\section{Result}

During the study period, we enrolled six patients with seven cases of dislocations beneath the heels. Our patients were all male with an average age of 38 years (range 36 and 47). In five cases, the etiology of the trauma was a motorcycle accident following an attempt to evict an obstacle with the notion of pivoting around a foot placed on the ground. This same mechanism was found in these five patients. In one case the etiology was a fall from height. The lesion was bilateral in one case (Figure 1). The trauma involved the right foot in 4 cases and the left foot in 3 cases. The average treatment time was 3 hours with extremes of 1 hour and 9 hours.

Clinical examination found deformity of the hindfoot in all patients. Ankle and foot edema was present in all of our patients. In one of our patients we observed a skin pain which evolved well after the operation. In the medial form the head of the talus was perceived laterally and the rest of the foot dislocated within. As for the lateral variety, the head of the talus was perceived medially and the rest of the foot dislocated outside. An open dislocation fracture with enucleation of the talus was noted (Figure 1). Sudetal dislocation was associated with head trauma in one patient.

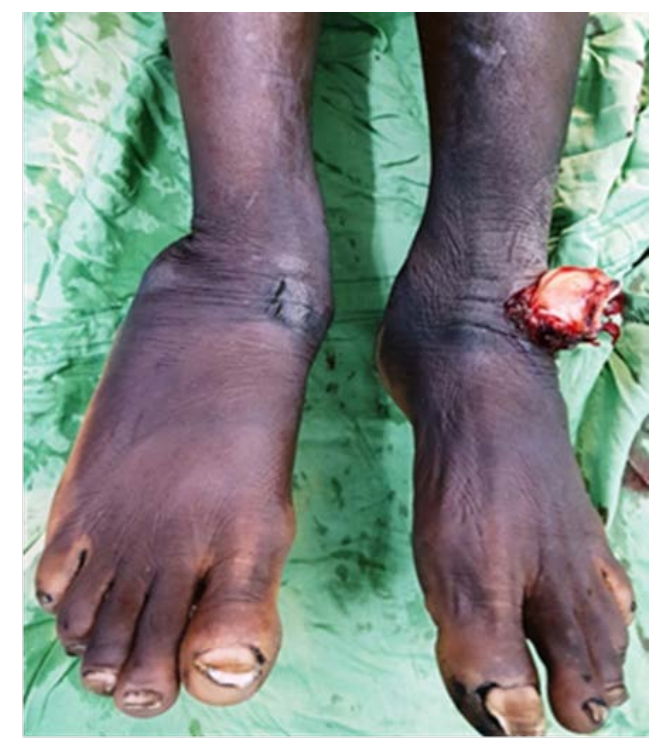

Figure 1. Initial clinical aspect of a bilateral luxation fracture under the talar closed on the right and open type II of GA on the left with enucleation of the talus. 
$\mathrm{X}$-rays of the foot and ankle, front and profile, allowed these dislocations to be broken. It was medial in 6 cases and lateral in one case.

We had observed three cases of pure talar dislocation. In four cases they were associated with bone lesions: talus fracture ( 2 cases) a medial malleolus fracture ( 1 case) (Figure 2), a bimaleolar fracture and second metatarsal fracture (1 case), could not be performed in our patients due to its unavailability.

The treatment was orthopedic in 2 cases and surgical in 5 cases. This surgery was indicated after failed attempts to reduce dislocation by external maneuver and in the form associated with a skin opening. In all cases, a plastered boot was made and kept for 6 weeks. In surgical treatment, the ankle approach was anterior (Figure 3).

The reduction was followed by a racking-in (Figure 4).

The post-operative suites were interspersed with superficial suppuration of the operative wound in one case. The pins were removed in the sixth post-operative week. The functional result according to the score of the American Orthopedic Foot and Ankle Society (AOFAS) was excellent in 2 cases; good 2 cases, fair 2 cases an $\mathrm{d}$ poor 1 case. The average length of hospital stay was 4 days.

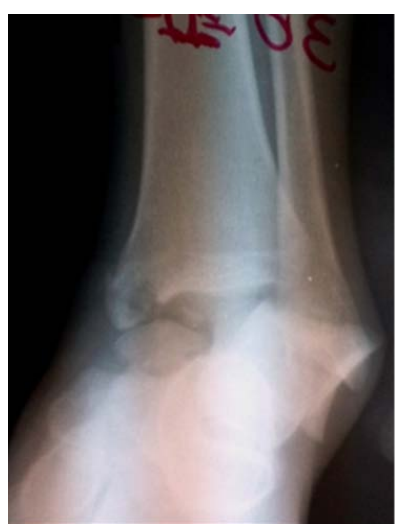

(a)

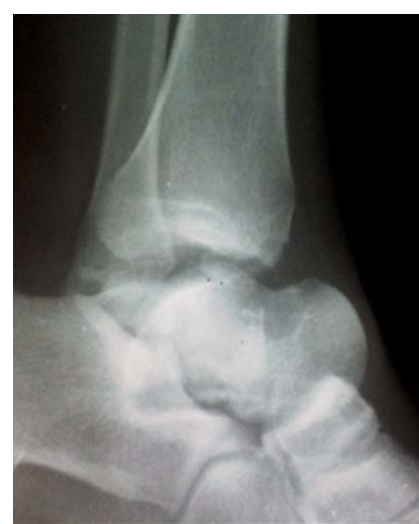

(b)

Figure 2. (a) and (b): Standard front (a) and side (b) radiographs of the left foot demonstrated a fracture of the talus and medial malleolus associated with subtalar dislocation.

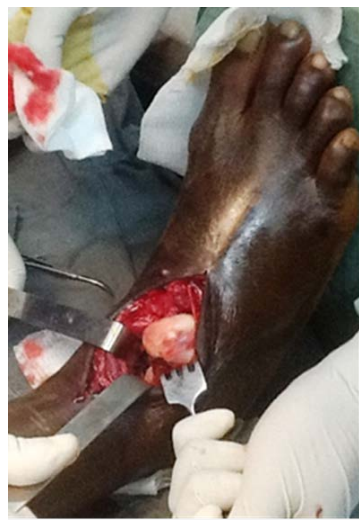

(a)

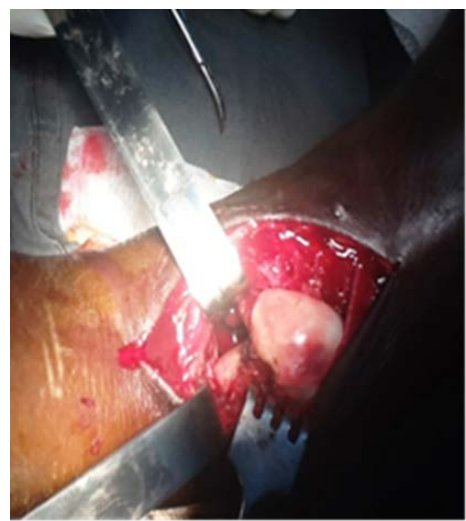

(b)

Figure 3. (a) and (b): Peroperative aspect showing talo-navicular dislocation. 


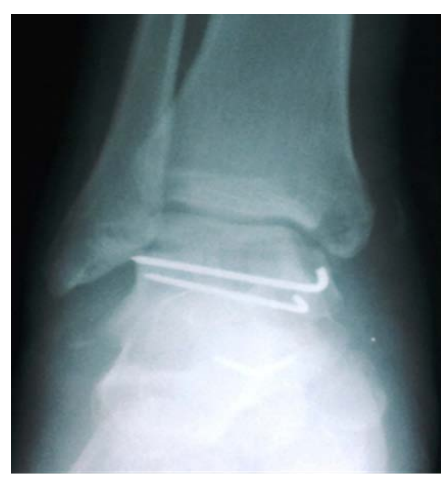

(a)

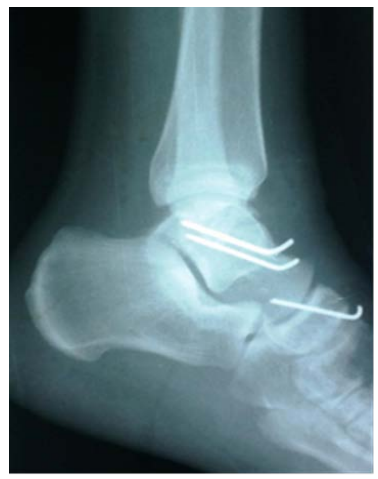

(b)

Figure 4. (a) and (b) On the postoperative radiographic image of the front of the foot (a), we visualize the restitution of the anatomical reports at the level of the subtalar joint. On the profile post-operative radiographic image (b) we can see the reduction in talo-navicular dislocation and the satisfactory reduction in the fracture of the talus.

\section{Discussion}

Our patients were young adult males who were victims of a traffic accident. This epidemiological profile can be superimposed on that of the literature [11] [12]. In our series, medical dislocation was the most common ( 6 cases). This variety is the most frequent and the most described in the literature [1] [12]. In $80 \%-85 \%$ of cases, the foot moves medially, bringing with it the calcaneus. The head of the slope remains prominent on the dorsolateral face. The lateral form is less frequent and represents $15 \%-20 \%$ of cases [13] [14]. It is important to distinguish the two forms because the causal mechanism, the reduction method and the long-term prognosis are different [13] [14].

The inversion of the foot is at the origin of the medial luxation while the eversion produces the lateral form. The powerful calcaneal-navicular ligament resists the inversion or eversion force which dissipates through the weak talo-navicular and talo-calcaneal. The rupture of these two ligaments promotes the displacement of the calcaneus, the navicular bone and the entire distal part of the bones of the foot either medially or laterally [14] [15] (Figure 5).

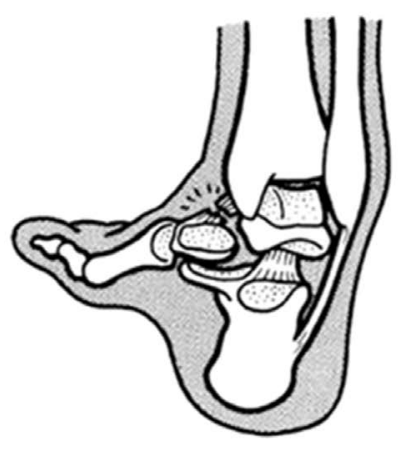

(a)

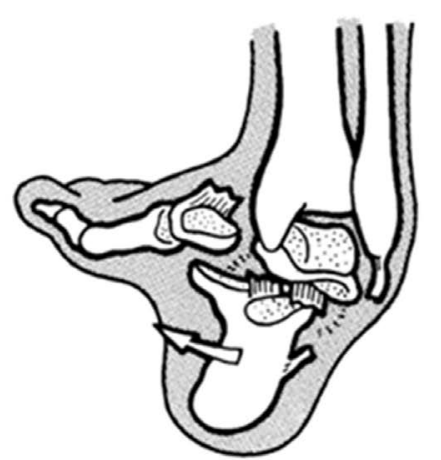

(b)

Figure 5. Medial subtalar dislocation results from a forced inversion of the foot. (a) 1st time: rupture of the dorsal talo-navicular ligament; (b) 2nd step: elongation or disinsertion of the interosseous talo-calcaneal ligament. 
We observed a case of bilateral dislocation. This bilaterality constitutes a rare entity, very little described in the literature.

The deformities of the foot and ankle observed in our series were consistent with the varieties of dislocation encountered. The ankle and foot edema observed in our patients is linked to the delay in taking which was on average 3 hours (range 1 and 9 hours). The skin pain observed in one of our patients progressed well postoperatively. According to Tucker et al, this skin pain is due to the pressure of the head of the slope which is prominent and palpable under the skin of the medial aspect of the foot [16].

Vascular-nerve damage, skin opening, malleolar and talus fractures are frequently described in the literature as lesions associated with dislocation [16] [17] [18] [19]. They witness the violence of the trauma. We had observed only one open dislocation case. These subtalar dislocations are associated with a skin opening in $10 \%$ to $40 \%$ of cases [20]. These skin openings are more frequent in lateral forms and in trauma with high velocity [20].

No vascular-nerve damage was detected. Monson and Ryan [21] reported a case with involvement of the posterior tibial artery and bruising of the nerve. Ischemia of the foot is more common in lateral dislocation because the posterior tibial artery is eased at the level of the talus [22]. Compression, stretching, or rupture of a vascular-nerve bundle can lead to abolition of the posterior and/or tibial pulse, and sensitivity disturbances.

Standard x-rays of the foot and ankle confirmed our diagnoses of dislocation and objectified associated bone lesions which were present in 5 cases. Associated osteocartilaginous lesions are frequently described in the literature, especially in lateral dislocations [19] [22]. They were not found in our series during intraoperative exploration due to the unavailability of CT scans.

We were able to urgently reduce 2 cases of medial variety by external maneuver. The other 5 cases, including a lateral form following the failure of your reduction attempt or skin opening, required a reduction in the sky. Several authors recommend the reduction of this dislocation in an emergency, which makes it possible to avoid necrosis of the underlying skin, to reduce the risk of irreducibility and to obtain a better functional result [12] [18] [21]. The keystone of treatment is rapid and gentle reduction under general or locoregional anesthesia [23]. Approximately $10 \%$ of medial dislocations and $15 \%$ to $20 \%$ of lateral forms cannot be reduced by external maneuver. The interposition of tissue (posterior tibial, long flexor of the hallux) and bone fragments have been identified as factors preventing reduction [14]. Reduction must always be followed by radiological monitoring to see the accuracy of reduction, look for associated fractures and exclude a diastasis between the medial malleolus and the talus, witness to damage to the deltoid ligament.

Like some authors, we had plastered for 6 weeks. For surgically reduced forms, pin stabilization was associated. This method is suspected of stiffening but is a guarantee against instability [22]. In our series we observed a case of superficial 
infection of the operating site which has progressed favorably, these infectious complications are frequent if the dislocation is open [24].

The 2 cases of excellent result concerned the pure forms reduced by external maneuver and immobilized by plastered boot. The high frequency of associated fractures in our series ( 5 cases), the irreducibility in some cases by external maneuver and the suppuration of the operating site would be our elements of poor prognosis. These different factors could explain our good, fair and poor results. Indeed the prognosis is good in simple dislocations without associated fracture and easily reduced by external maneuver [1]. According to Lancaster et al., the prognosis is poor when dislocation is associated with soft tissue damage, skin opening, extra-articular or joint fractures, infections, lateral dislocation, neglected dislocation and osteonecrosis [23].

In our series we noted 3 cases of osteoarthritis ( 2 subtalar cases and one talo-crural case). Ankle stiffness was observed in 3 patients. In fact, osteoarthritis, very common in certain series ( $25 \%$ to $60 \% \mathrm{~d}$ ), affects both the subtalar joint and that of Chopart, even the talo-crural joint [9] [16] [22]. According to Rammelt S [9] the presence of osteoarthritis could be explained by cartilaginous contusion, subchondral ischemia and mechanical dysfunction by injury of the ligament in hedge. However, stiffness and osteoarthritis predominate at the subtalar joint [9] [12] [24].

\section{Conclusion}

Subtalar dislocation is a rare and serious lesion of the posterior tarsus. Its diagnosis is guided by clinical and imagery which identifies the associated osteocartilaginous lesions. Its management is done urgently and consists of a reduction in dislocation followed by a plastered compression. The prognosis depends on the speed of treatment and the severity of the associated lesions.

\section{Conflicts of Interest}

The authors declare no conflicts of interest regarding the publication of this paper.

\section{References}

[1] Hoexum, F. and Heetveld, M.J. (2014) Subtalar Dislocation: Two Cases Requiring Surgery and a Literature Review of the Last 25 Years. Archives of Orthopaedic and Trauma Surgery, 134, 1237-1249. https://doi.org/10.1007/s00402-014-2040-6

[2] Broca, P. (1853) Dissertation on Subtalar Dislocations. Bulletins et mémoires de la Société de chirurgie de Paris, 3, 566-656.

[3] Jerome, J.T.J. (2008) Antero-Lateral Subtalar Dislocation. Foot and Ankle Surgery, 14, 36-39. https://doi.org/10.1016/j.fas.2007.08.002

[4] Zimmer, T.J. and Johnson, K.A. (1989) Subtalar Dislocations. Clinical Orthopaedics, 238, 190-194. https://doi.org/10.1097/00003086-198901000-00027

[5] Wagner, R., Blattert, T.R. and Weckbach, A. (2004) Talar Dislocations. Injury, 35, 
SB36-SB45. https://doi.org/10.1016/j.injury.2004.07.010

[6] Tucker, D.J., Burian, G. and Boylan, J.P. (1998) Lateral Subtalar Dislocation: Review of the Literature and Case Presentation. The Journal of Foot and Ankle Surgery, 37, 239-247. https://doi.org/10.1016/S1067-2516(98)80118-X

[7] Bibbo, C., Lin, S.S., Abidi, N., et al. (2001) Missed and Associated Injuries after Subtalar Dislocation: The Role of CT. Foot \& Ankle International, 22, 324-328. https://doi.org/10.1177/107110070102200409

[8] Rammelt, S., Winkler, J., Grass, R., et al. (2006) Reconstruction after Talar Fractures. Foot and Ankle Clinics, 11, 61-84. https://doi.org/10.1016/j.fcl.2005.11.002

[9] Malgaigne, J.F. (1855) Treatise on Fractures and Dislocations. Baillére Édition, Paris, $1030-107$.

[10] Kitaoka, H.B., Alexander, I.J., Adelaar, R.S., Nunley, J.A., Myerson, M. and Sanders, M. (1994) Clinical Rating Systems for the Ankle, Hindfoot, Midfoot, Hallux and Lesser Toes. Foot \& Ankle International, 15, 349-353. https://doi.org/10.1177/107110079401500701

[11] Heppenstall, R.B., Farahvar, H., Balderston, R., et al. (1980) Evaluation and Management of Subtalar Dislocations. Journal of Trauma, 20, 494-497. https://doi.org/10.1097/00005373-198006000-00011

[12] Rammelt, S. and Goronzy, J. (2015) Subtalar Dislocations. Foot and Ankle Clinics, 20, 253-264. https://doi.org/10.1016/j.fcl.2015.02.008

[13] DeLee, J.D. and Curtis, R. (1982) Subtalar Dislocation of the Foot. The Bone \& Joint Journal, 64A, 433-437. https://doi.org/10.2106/00004623-198264030-00014

[14] Heppenstall, R.B., Farahvar, H., Balderston, R., et al. (1980) Evaluation and Management of Subtalar Dislocations. Journal of Trauma, 20, 494-497. https://doi.org/10.1097/00005373-198006000-00011

[15] Grantham, S.A. (1964) Medial Subtalar Dislocation: Five Cases with a Common Etiology. Journal of Trauma, 4, 845-849. https://doi.org/10.1097/00005373-196411000-00012

[16] Bibbo, C., Anderson, R.B. and Davis, W.H. (2003) Injury Characteristics and the Clinical Outcome of Subtalar Dislocations: A Clinical and Radiographic Analysis of 25 Cases. Foot \& Ankle International, 24, 158-163. https://doi.org/10.1177/107110070302400210

[17] Leitner, B. (1954) Obstacles to Reduction in Subtalar Dislocations. The Bone \& Joint Journal, 36A, 299-306. https://doi.org/10.2106/00004623-195436020-00008

[18] Asencio, G. (1994) Sprains and Dislocations of the Talus. The Pathology of the Subtalar Joint. Masson, Paris, 111-114.

[19] Merianos, P., Papagiannakos, K., Hatzis, A. and Tsafantakis, E. (1988) Peritalar Dislocation: A Follow-up Report of 21 Cases. Injury, 19, 439-442. https://doi.org/10.1016/0020-1383(88)90143-X

[20] Golner, J.L., Poletti, S.C., Gates, H.S., et al. (1995) Severe Open Subtalar Dislocations: Long-Term Results. The Bone \& Joint Journal, 77 A, 1075-1079. https://doi.org/10.2106/00004623-199507000-00015

[21] Monson, S.T. and Ryan, J.J. (1981) Subtalar Dislocation. The Bone \& Joint Journal, 63A, 1156-1158. https://doi.org/10.2106/00004623-198163070-00013

[22] Conesa, X., Barro, V., Barastegu, D., Batalla, L., Tomas, J. and Molero, V. (2011) Lateral Subtalar Dislocation Associated with Bimalleolar Fracture: Case Report and Literature Review. The Journal of Foot \& Ankle Surgery, 50, 612-615. 
https://doi.org/10.1053/j.jfas.2011.04.034

[23] Bohay, D.R. and Manoli, A. (1995) Subtalar Dislocations. Foot \& Ankle International, 16, 803-808. https://doi.org/10.1177/107110079501601212

[24] Christensen, S.B., Lorentzen, J.E., Krogsoe, O. and Sneppen, O. (1977) Subtalar Dislocation. Acta Orthopaedica Scandinavica, 48, 707-711.

https://doi.org/10.3109/17453677708994821 